\title{
Phase analysis of the first pass radionuclide angiocardiogram
}

\author{
S WALTON, P J ELL, P H JARRITT, R H SWANTON \\ From the Departments of Cardiology and Nuclear Medicine, Middlesex Hospital and Medical School, London
}

SUMMARY Fifty-two subjects with suspected coronary artery disease were studied by first pass radionuclide angiocardiography using a single crystal gamma camera. Phase analysis, a new technique which can detect ventricular regions with abnormal emptying patterns, was applied to the resulting left ventricular radionuclide angiocardiograms.

Eighteen turned out to be normal and in these subjects the variation of phase values in each of eight ventricular segments was established.

Thirty-four had coronary artery disease. By comparison with the normal subjects, 21 had at least one segment with an abnormally high phase value. Such high values were associated with low values of ejection fraction, as determined by a radionuclide technique, total occlusion of a major coronary artery and significant wall motion abnormalities on the radiographic ventriculogram. A comparison between phase analysis and wall motion on a regional basis disclosed progressively high phase values with increasing severity of wall motion abnormality. Particularly high values were found in association with ventricular aneurysm.

Phase analysis of first pass radionuclide angiocardiograms is a valid method for the detection of regional abnormalities of ventricular contraction in coronary artery disease.

Phase analysis is a recent development of radionuclide angiocardiography which can be used to detect and quantify regional abnormalities of ventricular contraction by the temporal characteristics of their emptying patterns. The results are displayed as an image of the intracardiac blood pool, colour coded in such a way that regions of delayed or paradoxical emptying are clearly distinguished from normal regions. The technique shows great promise but requires further validation. So far it has been applied only to studies performed after equilibration of an intravascular tracer. Such an approach necessitates imaging in the left anterior oblique projection to achieve spatial separation between the two ventricles. Furthermore, data accumulation time is long and background levels are low. Images of the left ventricle can be obtained during the first pass of a peripherally injected radionuclide bolus through the central circulation. Data accumulation time is short, background levels low, and the temporal separation of right and left heart transit permits right anterior oblique imaging to be performed. This latter aspect facilitates comparison with standard wall motion techniques which are best performed in this view. On the other hand, the opportunity for data collection in first pass studies is limited and the high count rates encountered place severe demands on conventional imaging devices.

The purpose of this study was firstly to show that phase analysis of the first pass radionuclide angiocardiogram can be performed with a modern single crystal gamma camera and, secondly, taking advantage of the facility for right anterior oblique imaging, to provide further validation for the technique of phase analysis by comparison with cardiac catheterisation data.

\section{Subjects and method}

Fifty-two subjects with suspected coronary artery disease were studied by first pass radionuclide angiocardiography. Forty-six were men whose ages ranged between 33 and 72 (mean 52). $740 \mathrm{MBq}$ of 
${ }^{99} \mathrm{Technetium}$ as pertechnetate were injected as a bolus into a medial antecubital fossa vein and flushed with 20 $\mathrm{ml}$ saline. The initial passage of tracer through the central circulation was detected by a General Electric IGE 400T large field of view gamma camera, fitted with a high sensitivity, parallel hole collimator and positioned over the precordium in the $30^{\circ}$ right anterior oblique projection. Data were recorded in list mode and analysed using the standard software of an Informatek Simis 3 computer. Electrocardiographic gating was used to identify individual cardiac cycles and a 16 frame representative cine cycle, which served as the basis for further analysis, was produced by summing data from six to 12 cycles during the left heart phase of bolus transit. Each display element from a 32 by 32 array which contained the heart was used to produce a 16 point curve by plotting the activity from that element for each frame of the representative cine cycle.

The variation of activity of such curves differs in both magnitude and timing in different areas of the display. Background elements show little variation. Atrial and ventricular elements usually show a distinct rise and fall of activity during the cardiac cycle, reflecting filling and emptying. The timing of this variation of activity differs between the two chambers as it may do in different regions of the ventricle. Curves showing less than $15 \%$ of the maximum variation were assumed to be those of background display elements and were excluded from further analysis.

For all remaining elements "phase" was calculated from the activity time curve. The mathematical derivation of "phase" has been described. 12 In general, it is true that curves with early emptying are assigned low values of phase. Progressively later emptying is associated with progressively higher phase. Each display element was colour coded according to phase, thus producing the phase image. A method of phase quantification on a regional basis was devised. Each left ventricular phase image was divided into eight overlapping $90^{\circ}$ segments centred on the centre of gravity of the phase image. Segment 1 included the anterobasal region, segments 2 to 4 the anterior wall, segments 4 to 6 the apex, and segments 6 to 8 the inferior wall and inferobasal region. Mean phase was calculated for each segment.

A region of interest was manually assigned to the left ventricle on the phase image. After background correction using a functional technique ${ }^{3}$ the variation of activity within this region during the representative cine cycle was used to calculate left ventricular ejection fraction.

Forty-four subjects underwent cardiac catheterisation. Coronary arteriography was performed in at least three views by either the Sones or Judkins technique. Left ventricular cine angiography was performed in the $30^{\circ}$ right anterior oblique projection and ventriculograms were analysed both subjectively (method 1) and by superimposition of end-diastolic and end-systolic outlines with subsequent estimation of minor axis shortening (method 2). Wall motion was considered in anterior, apical, and inferior regions and was classified as normal, hypokinetic, akinetic, or paradoxical. The term aneurysm was applied either to a localised area of paradoxical wall motion or to an extensive area of dilatation and akinesis.

Catheterisation was performed within 48 hours of the radionuclide study. There were no significant alterations of treatment between the two procedures. The subjects fell into 3 groups-

Group A: normal $(\mathrm{n}=18)$. Either normal catheterisation findings $(n=10)$ or normal rest and stress electrocardiography: without catheterisation $(\mathrm{n}=8)$.

Group B: coronary disease but no total occlusion of a major coronary artery $(n=11)$.

Group $C$ : at least one major coronary artery totally occluded $(n=23)$.

Normal segmental phase values were defined as those that were less than three standard deviations from the mean value for each segment for the group A subjects. Segmental values for groups $b$ and $c$ subjects were plotted against the normal range. The maximum degree to which phase was abnormal (that is the difference between the observed value and the upper limit of the normal range) in anterior, apical, and inferior regions was calculated.

Phase values were assessed in the light of ejection fraction values, the presence of total occlusion of a major coronary artery, and radiographic wall motion evaluation.

Table 1 Abnormal phase values and ejection fractions for group $B$

\begin{tabular}{lllll}
\hline No. & LVEF & \multicolumn{3}{l}{ Abnormal phase } \\
\cline { 3 - 4 } & & Anterior & Apical & Inferior \\
\hline 1 & 45 & & & \\
2 & 53 & & & \\
3 & 76 & 15 & 15 & \\
4 & 62 & & & \\
5 & 46 & & & \\
6 & 54 & & & \\
7 & 37 & 15 & & \\
8 & 66 & & & \\
9 & 58 & 15 & 5 \\
10 & 55 & & \\
11 & 71 & & \\
\hline
\end{tabular}

All had normal ventriculograms except case 7 who had inferior hypokinesis. LVEF, left ventricular ejection fraction. 
Table 2 Abnormal phase values, ejection fractions, and catheterisation data in group $C$

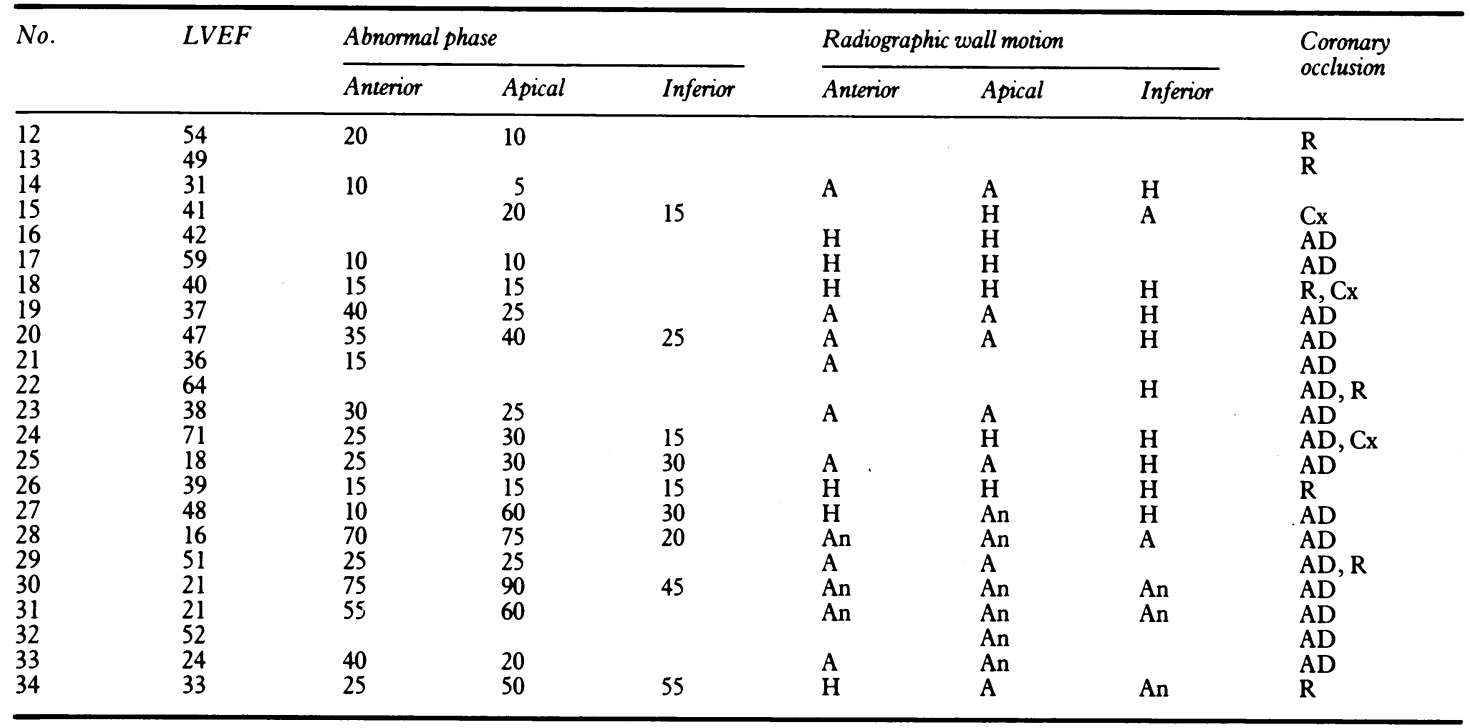

$\mathrm{R}$, right; $\mathrm{Cx}$, circumflex; $\mathrm{AD}$, anterior descending arteries; $\mathrm{A}$, akinesis; $\mathrm{H}$, hypokinesis; $\mathrm{An}$, aneurysm.

Table 3 Comparison of two methods of analysis of the radiographic ventriculograms

\begin{tabular}{lrrll}
\hline & Normal & Hypokinesis & Akinesis & Aneurysm \\
\hline Method 1 & & & & \\
$\quad$ Normal & 40 & 4 & 5 & \\
$\begin{array}{l}\text { Hypokinesis } \\
\text { Method 2 }\end{array}$ & 3 & 20 & 5 & 2 \\
$\quad \begin{array}{l}\text { Akinesis } \\
\text { Aneurysm }\end{array}$ & 3 & 9 & 13 \\
\hline
\end{tabular}

\section{Results}

The maximum instantaneous count rate during the left heart phase of bolus transit was always less than 60000 per second. At these levels the count losses of this gamma camera are less than $10 \%$.

End-diastolic left ventricular counts after background correction were between 5000 and 32500 (mean 18500).

Tables 1 and 2 show the results of phase analysis, ejection fraction estimation, and cardiac catheterisation for groups B and C, respectively, and Table 3 a comparison of the two methods of analysis of the radiographic ventriculograms.

Fig. 1 shows the regional variation of phase values in the group A subjects and Fig. 2 a normal phase image. Figs. 3 and 4 show the results of phase analysis and radiographic ventriculography in two group C subjects. Regional phase values are compared to wall motion analysis in Fig. 5 and the maximum value of abnormal phase to ejection fraction in Fig. 6 .

Twelve subjects with coronary artery disease had normal radiographic ventriculograms (left ventricular ejection fraction (LVEF) $57 \pm 10 \%$, mean \pm SD). Nine had normal phase images. The remainder had only minor abnormalities of phase. Twenty-two had abnormal ventriculograms and of these all but four had abnormal phase values. In three of the latter the radiographic abnormality was localised hypokinesis. The subjects with abnormal ventriculograms consisted of seven (LVEF $49 \pm 13 \%$ ) with regional or general hypokinesis, eight (LVEF $37 \pm 13 \%$ ) with localised akinesis, and seven (LVEF $30 \pm 10 \%$ ) with ventricular aneurysm.

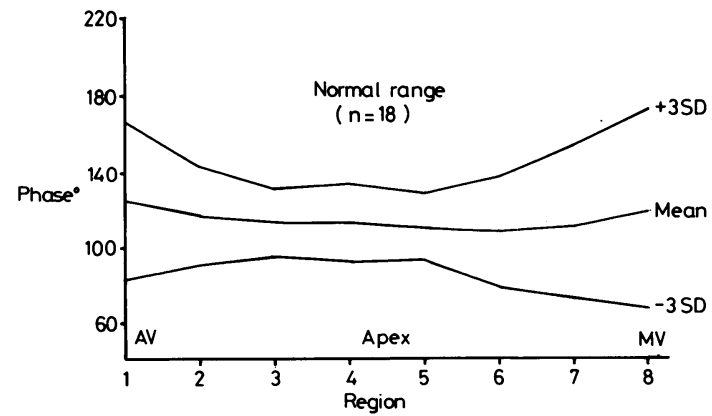

Fig. 1 Segmental phase values for group $A . A V$, aortic valve; $M V$, mitral valve. 
(a)

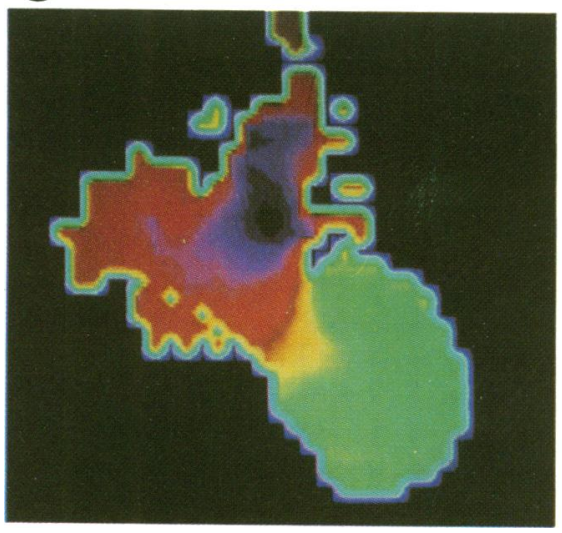

(b)

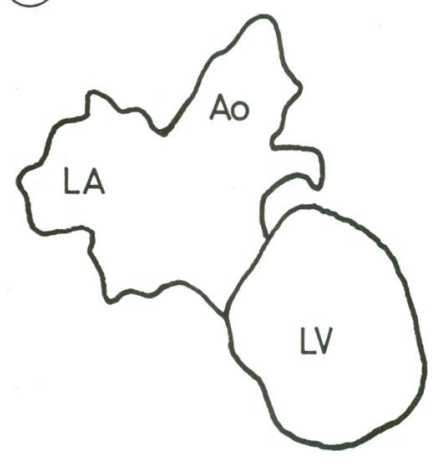

(a)

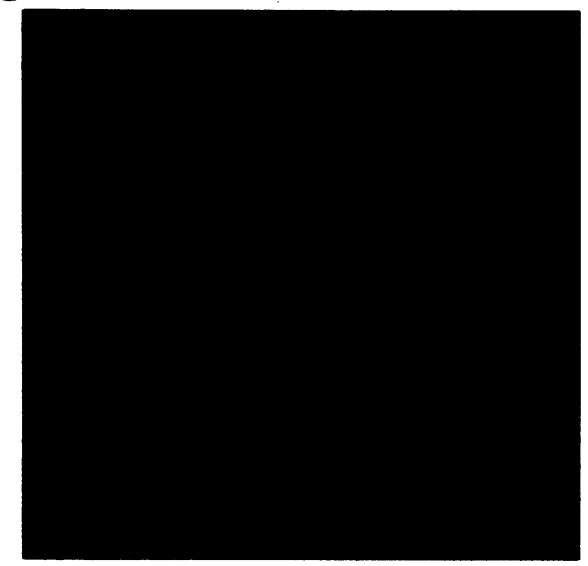

(b)

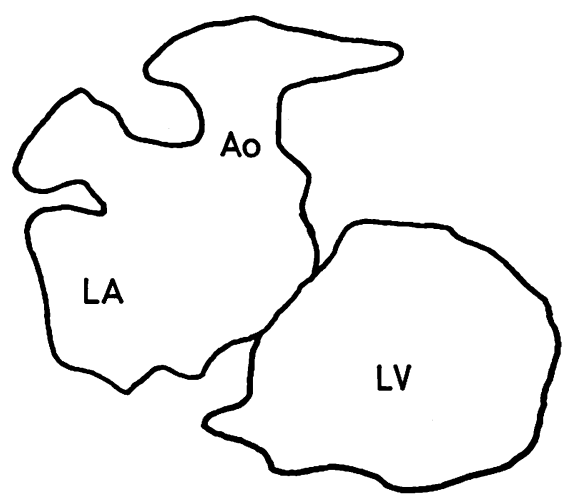

(c)

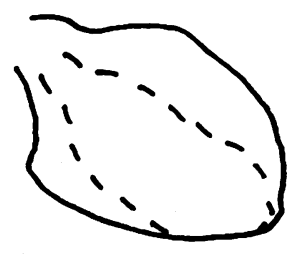

Fig. 2 (a) Normal phase image. (b) Line diagram. The left ventricle $(L V)$ empties relatively early in the cardiac cycle and is therefore a region of low phase, here shown green. The left atrium $(L A)$ and aorta $(A o)$ empty late in the cycle and are therefore regions of high phase, shown as red and purple, respectively. Note intermediate phase values, yellow, where the low phase ventricle overlaps the high phase atrium.

Fig. 3 (a) Phase image after myocardial infarction. (b) Line diagram. (c) Superimposed end-diastolic (_) and end-systolic (_ _ - ) outlines from the ventriculogram. (d) Plot of abnormal segmental phase values (_- _) against the upper limit of the normal range (__ ). Note yellow intermediate phase values at the base (within normal range) and in the inferoapical region (abnormal). $L V$, left ventricle; $L A$, left atrium; Ao, aorta.

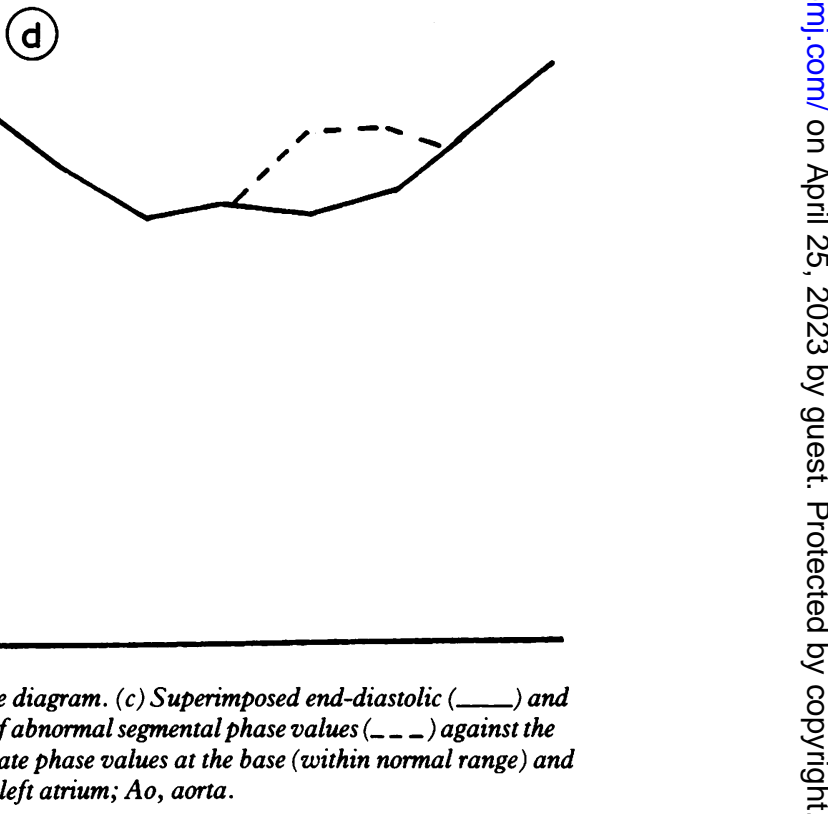


(a)

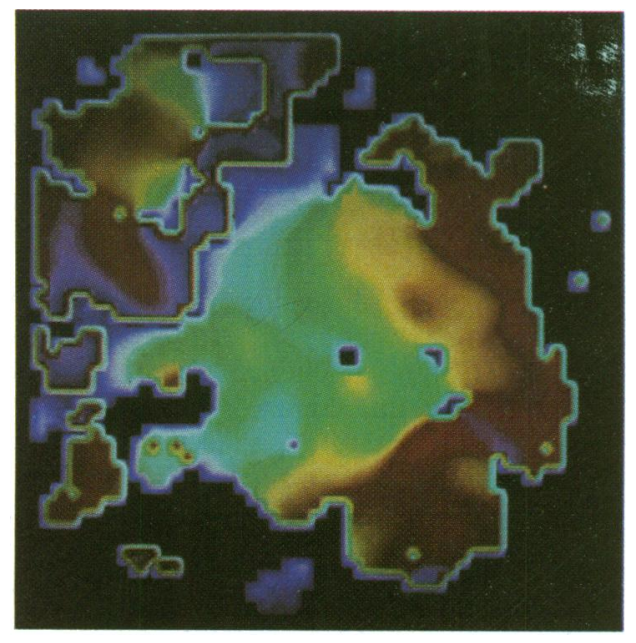

(c)
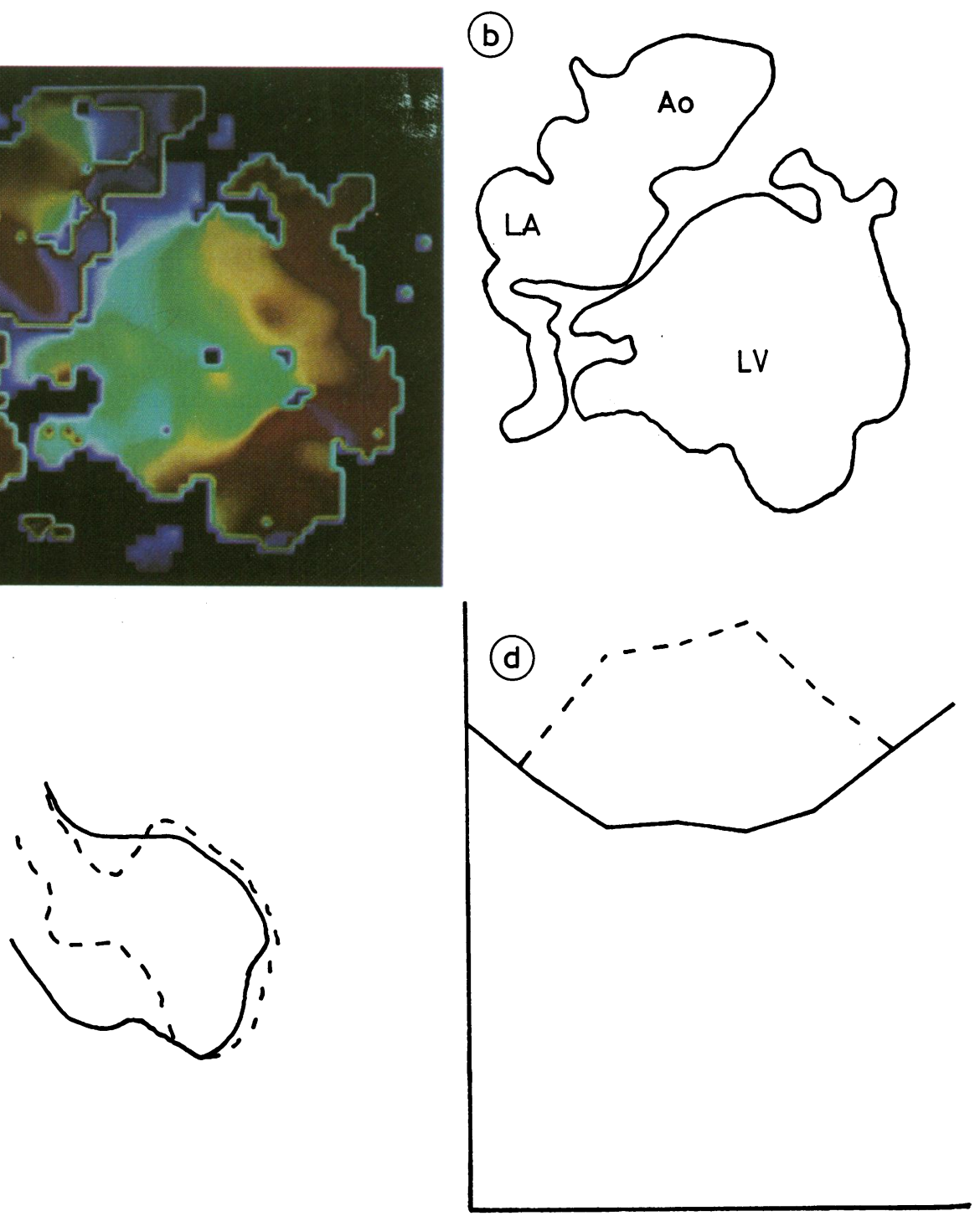

Fig. 4 (a) Phase image from a subject with a large left ventricular aneurysm. (b) Line diagram. (c) Superimposed end-diastolic (__ and end-systolic (_ _- ) outlines from the ventriculogram. (d) Plot of abnormal segmental phase values (_ _ - against the upper limit of the normal range (__ ). Note large area of high phase (red) involving anterior, apical, and inferior regions of the left ventricle. $L V$, left ventricle; $L A$, left atrium; $A o$, aorta.

\section{Discussion}

Phase analysis, first described by Adam et al., ${ }^{4}$ is a potentially important development of radionuclide angiocardiography. It can facilitate region of interest selection, thus improving the accuracy of ejection fraction estimation and the measurement of ventricular volumes. ${ }^{5}$ It can show abnormal patterns of ventricular activation ${ }^{2}$ and it can detect and quantify the extent of abnormalities of ventricular contraction produced by coronary artery disease. ${ }^{6}$ Furthermore, it can help in the preoperative evaluation of patients with ventricular aneurysm. ${ }^{6}$ Precise edge definition, a major disadvantage with radionuclide wall motion studies, ${ }^{7}$ is not essential and, in contrast to other techniques, meaningful information is obtained from all regions of the image and not just from a small rind around the perimeter. 


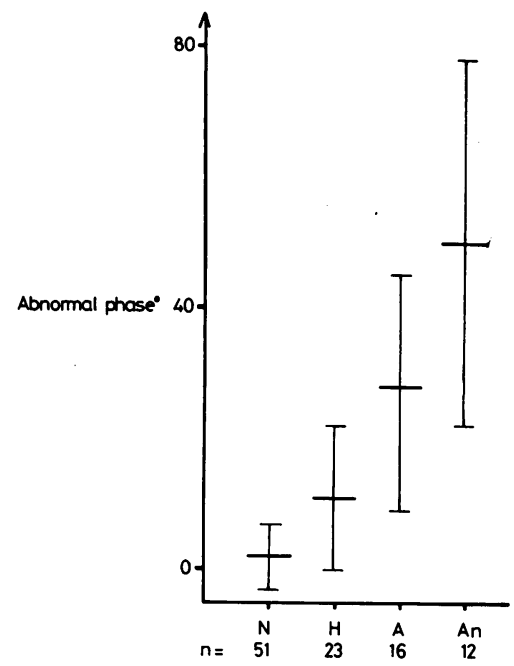

Fig. 5 Regional comparison of abnormal phase values (mean $\pm S D$ ) with radiographic wall motion. Three regions per ventricle. Groups $B$ and $C$. $N$, normal; $H$, hypokinetic; $A$, akinetic; $A n$, aneurysmal wall motion. The difference between the mean phase value of those regions with normal radiographic wall motion and that of those with hypokinesis, akinesis, or aneurysm was statistically highly significant $(p<0.005$ in each case).

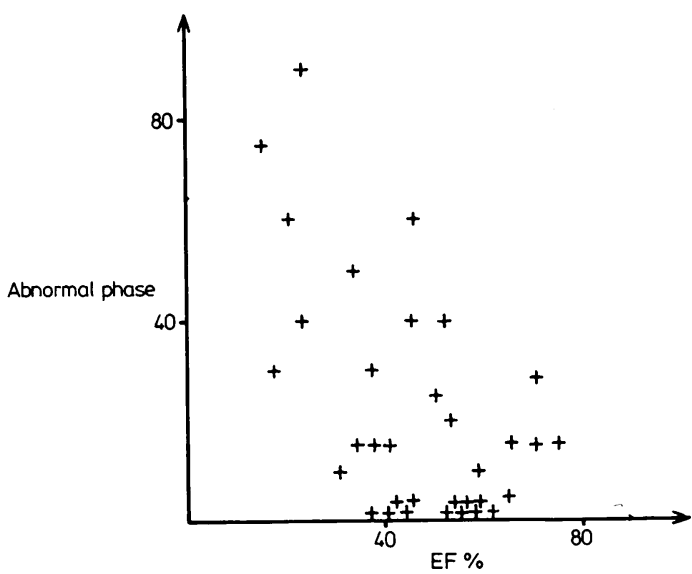

Fig. 6 Comparison of maximum value of abnormal phase with ejection fraction. Groups $B$ and $C$.

On the other hand, there are a number of theoretical and practical difficulties with the technique. The method as described uses only the fundamental frequency of the Fourier transform and assumes that the heart rate is the base frequency. Thus changes in the length of diastole consequent upon a change in heart rate will affect the value of phase. Though of little importance when comparing different regions with the ventricle, it could well have a significant effect on $\overrightarrow{\vec{s}}$ interpatient and rest exercise comparisons. Attempts $\overrightarrow{0}$ have been made to correct this by application of a $\overline{0}$ variable base frequency derived from the length of $\frac{\bar{p}}{7}$ systole as measured from the left ventricular activity time curve. ${ }^{89} \mathrm{~A}$ further difficulty arises from the interpretation of abnormal phase values which are, after all, an attempt to define a curve by a single value. The production of similar parametric images showing the regional distribution of stroke volume (amplitude image) or regional ejection fraction is helpful in this respect to distinguish, for example, high phase values resulting from conduction abnormality and those resulting from infarction.

Of more practical importance are problems arising from the use of a $15 \%$ cut off to define the edge of the 윽 ventricle. Certain regions of abnormal ventricles may fall below the cut off point and therefore be assigned a value of zero phase. This may affect the value of mean regional phase and, when taken together with the already reduced spatial resolution induced by the limited opportunity for data collection, can result in poor image quality in abnormal ventricles.

Finally, absorption of radiation from deeper by more superficial regions of the ventricle leads to poor sensitivity for "posterior" lesions and movement of the heart as a whole has an unknown effect on phase values. The latter may be less of a problem than for techniques such as wall motion analysis because the exact edge position is not crucial and emptying may, to some extent, compensate for cardiac rotation. In this respect it is interesting to note that the normal subjects did not manifest spurious regions of high phase.

Thus, despite its many advantages, phase analysis requires further validation. The most straightforward approach, and one adopted in this study, is to establish a normal range for subsequent comparison of experimental data. Fig. 1 shows the range of phase values found in the normal subjects. The upper limit varies in different segments, a consideration that prevents the adoption of a single value as the upper limit of normal. $\frac{\text { ? }}{3}$ Instead, the degree to which phase is abnormal must be $\rightarrow$ determined for each segment independently. The variation observed in both basal regions probably o reflects overlap with left atrium. As the latter have high phase values regions of overlap show intermediat values.

In order to provide further validation of phas analysis comparison was made with conventiona techniques. There is a good relation between abnorma phase values and total occlusion of a major coronar artery. Though minor abnormalities of phase did occu in three group B subjects the more pronounce abnormalities of phase were only found in group 1 subjects. Thus, it is likely that abnormal phase value reflect ventricular damage secondary to total occlusio 
of a major coronary artery.

Ejection fraction is not a sensitive indicator of regional ventricular disease because of compensation by healthy regions. Nevertheless, it is a good index of overall ventricular contraction and there is a trend towards increasing abnormality on the phase image and decreasing ejection fraction (Fig. 6). Thus distinct abnormality of phase is found in severely damaged ventricles.

The comparison of new techniques for the detection of regional abnormalities of ventricular contraction with standard techniques is by no means a straightforward matter, mainly because of the imperfections of the latter. Wall motion abnormalities can be detected from the radionuclide angiocardiogram but difficulties with edge definition ${ }^{7}$ leave such studies open to considerable criticism. Furthermore, they have only achieved their limited degree of acceptance by correlation with the radiographic ventriculogram. For these reasons and also because the radiographic approach is still widely assumed to be the accepted standard, radiographic ventriculography was chosen in this study. Subjective analysis of such studies is well known to be of limited reproducibility. Objective techniques differ in results depending on whether they correct for cardiac motion and, if so, how they do it, and whether or not they use a single image for end-systole. In this study subjective analysis and superimposition of enddiastolic and end-systolic images were performed. Table 3 shows that there were differences between the two but these were not major. Accordingly, the more objective approach was used for the comparison. That this was a reasonable technique to use is supported by the trend for decreasing ejection fraction values with increasing abnormality of wall motion. Taking these factors into account together with the fact that the amplitude of wall motion and its timing are independent variables minor differences between wall motion and phase analysis are to be expected. The overall trend towards increasing abnormality of phase with increasing abnormality of wall motion (Fig. 5) is unmistakable and provides good evidence that phase analysis can be used not only to detect regional abnormalities of contraction but also to quantify them.

Finally, these results confirm that meaningful information about regional left ventricular function can be obtained from first pass studies if modern single crystal gamma cameras are used. ${ }^{10-13}$

\section{Conclusion}

First pass studies of the left ventricle performed with modern single crystal gamma cameras are suitable for phase analysis. Normal subjects have generally higher phase values at the base of the ventricle than at the apex, a factor that must be taken into account when interpreting the phase image.

Abnormal phase values are found in association with total occlusion of a major coronary artery, low values of ejection fraction, and significant wall motion abnormalities on the radiographic ventriculogram. Furthermore, there is a progressive rise in the abnormality of phase with increasing severity of wall motion abnormality from the radiographic study.

Phase analysis of the first pass radionuclide angiocardiogram is a non-invasive technique that can be used to detect and quantify regional abnormalities of ventricular contraction in coronary artery disease.

The work was supported by a grant from the Sir Jules Thorn Charitable Trust.

\section{References}

1 Bossuyt A, Deconinck F, Lepoidre R, Joncker M. The temporal Fourier transform applied to the functional isotope image. Information processing in medical imaging. Proceedings of 6th international conference INSERM 1979; 88: 397-408. (ISBN 2-85598-191-3.)

2 Links JM, Douglass KH, Wagner HN Jr. Patterns of ventricular emptying by Fourier analysis of gated bloodpool studies. F Nucl Med 1980; 21: 978-82.

3 Goris ML, Briardet PA, Hoffer E. Automation and operator independant data processing of cardiac and pulmonary function: role, methods and results. Information processing in medical imaging. Proceedings of 6 th international conference. INSERM 1979; 88: 477-88. (ISBN 2-85598-191-3.)

4 Adam WE, Tarkowska A, Bitter F, Stauch M, Geffers H. Equilibrium (gated) radionuclide ventriculography. Cardiovasc Radiol 1979; 2: 161-73.

5 Walton S, Jarritt PH, Ell PJ. Improved reproducibility of ejection fraction estimation utilising the phase image. Use of the technique to determine the heart's response to isometric exercise. In Nuclear Medicine. Stuttgart: Schattauer Verlag 1980: 22P-224. (ISBN 3-7945-0808-4.)

6 Walton S, Yiannikas J, Jarritt PH, Brown NJG, Swanton RH, Ell PJ. Phasic abnormalities of ventricular emptying in coronary artery disease. $\mathrm{Br}$ Heart $\mathrm{f} 1981$; 46: 245-53.

7 Chang W, Henkin RE, Hale DJ, Hall D. Methods for detection of left ventricular edges. Semin Nucl Med 1980; 10: 39-53.

8 de Graff CN, van Rijk PP. Improved cardiac phase/ amplitude functional imaging. Nuclear Medicine Communications 1980; 1: 19-22.

9 Vos PH, Vossepoel AM, Beekhuis H, Pauwels EKG. Characterisation of ventricular function by temporal Fourier transform of gated blood pool studies. Nuclear Medicine Communications 1980; 1: 10-7.

10 Jengo JA, Mena I, Blaufuss A, Criley JM. Evaluation of left ventricular function (ejection fraction and segmental wall motion) by single pass radioisotope angiography. Circulation 1978; 57: 326-32. 
11 Hecht HS, Mirell SG, Rolett EL, Blahd WH. Leftventricular ejection fraction and segmental wall motion by peripheral first-pass radionuclide angiography. $\mathcal{F ~} \mathrm{Nucl}$ Med 1978; 19: 17-23.

12 Pachinger O, Ogris E, Probst P, Joskowics G, Sochor H, Kaindl F. Assessment of left ventricular performance and myocardial viability using quantitative radioisotope techniques. Br Heart f 1979; 42: 205-13.

13 Walton S, Donaldson EA, Rowlands DJ, Shields RA,
Testa HJ, Wrigley C. Regional left ventricular wall motion from first pass radionuclide angiocardiography using an Anger camera. An experimental and clinical study. Br Heart f 1980; 44: 518-23.

Requests for reprints to Dr S Walton, Department of Cardiology, The Middlesex Hospital, Mortimer Street, London WIN 8AA. 